\title{
Regulation of glycogen synthase activity and phosphorylation by exercise
}

\author{
Jakob N. Nielsen and Jørgen F. P. Wojtaszewski* \\ Copenhagen Muscle Research Centre, Institute of Exercise and Sport Sciences, University of Copenhagen, \\ Copenhagen, Denmark
}

\begin{abstract}
Glycogen synthase (GS) catalyses the rate-limiting step of UDP-glucose incorporation into glycogen. Exercise is a potent regulator of GS activity, leading to activation of GS immediately after exercise promoting glycogen repletion by mechanisms independent of insulin. The incorporation of UDP-glucose is energy demanding, and during intense exercise GS is deactivated, diminishing energy utilization but also increasing the potential for glycogen breakdown. An apparent activation of GS is observed during moderate exercise, which could be considered as a potential waste of energy, although the cellular capacity for glycogen breakdown is considerably higher than that for glycogen synthesis. The understanding of this complex regulation of GS activity in response to exercise is just at its beginning. In the present review potential mechanisms by which exercise regulates GS activity are described, factors that may promote GS activation and factors that may deactivate GS are discussed, pointing to the view that GS activity during exercise is the result of the relative strength of these opposing factors.
\end{abstract}

Exercise: Contraction: Skeletal muscle: Glycogen synthase: Phosphorylation

Glycogen is an essential metabolic substrate during exercise, even at fairly low work intensities. After a glycogen-depleting stimulus, e.g. exercise, the rise in plasma insulin induced by a carbohydrate-rich meal directs a substantial part of the glucose towards repletion of the glycogen stores. This process occurs by insulin-dependent and insulin-independent increases in both glucose uptake and glycogen synthesis. A key enzyme in glycogen synthesis is glycogen synthase (GS), which catalyses the incorporation of UDP-glucose into glycogen. GS activity is regulated allosterically by glucose-6-phosphate (G6P), as well as by reversible phosphorylation and dephosphorylation leading to inactivation and activation of GS respectively. Thus, activation of GS may take place through inactivation of kinases phosphorylating GS and by activation of phosphatases dephosphorylating GS. Several of these upstream enzymes have been identified, although their relative importance in the regulation of GS activity in vivo has not been fully clarified. In general, phosphorylation decreases GS activity by causing an increase in the $K_{m}$ for the substrate UDP-glucose and an increase in the $K_{a}$ for G6P (Roach \& Larner, 1976; Roach et al. 1976). GS is subject to phosphorylation at nine or more sites. Two sites, sites 2 and $2 \mathrm{a}$, corresponding to $\operatorname{Ser}^{7}$ and $\operatorname{Ser}^{10}$ on GS from rabbit skeletal muscle, are located near the $\mathrm{NH}_{2}$ terminus. At least seven sites (sites $3 a-c, 4,5,1 a-b)$ are located in the $\mathrm{COOH}$-terminal 100 residues of GS. Phosphorylation of sites $2,2 \mathrm{a}, 3 \mathrm{a}$ and $3 \mathrm{~b}$ generally decreases the activity more than phosphorylation of the remaining sites that have minor or no effect on GS activity in vitro (for review and further references, see Roach, 2002). Notably, initial phosphorylation of site 5 by casein kinase 2 creates a recognition motif for GS kinase 3 (GSK3), which then sequentially phosphorylates sites $4,3 \mathrm{c}, 3 \mathrm{~b}$ and $3 a$. This phenomenon has been termed hierarchal phosphorylation (see Fig. 1), which has also been demonstrated for the $\mathrm{NH}_{2}$-terminal sites of GS where modification of site 2 by protein kinase A (PKA), 5'AMP-activated protein kinase (AMPK) or other kinases is a prerequisite for phosphorylation of site $2 \mathrm{a}$ by casein kinase 1 .

\footnotetext{
Abbreviations: AICAR, 5'-aminoimidazole-4-carboxamide-1- $\beta$-D-ribofuranoside; AMPK, 5'AMP-activated protein kinase; CaMK, Ca ${ }^{2+} / \mathrm{calmodulin}^{-}$ dependent kinase; G6P, glucose-6-phosphate; $\mathrm{G}_{\mathrm{M}}$, glycogen-associated PP1 subunit; GS, glycogen synthase; GSK3, GS kinase 3; PhK, glycogen phosphorylase kinase; PKA, protein kinase A; PP1, protein phosphatase 1 .

*Corresponding author: Dr J. F. P. Wojtaszewski, fax +45353216 00, email jwojtaszewski@aki.ku.dk
} 


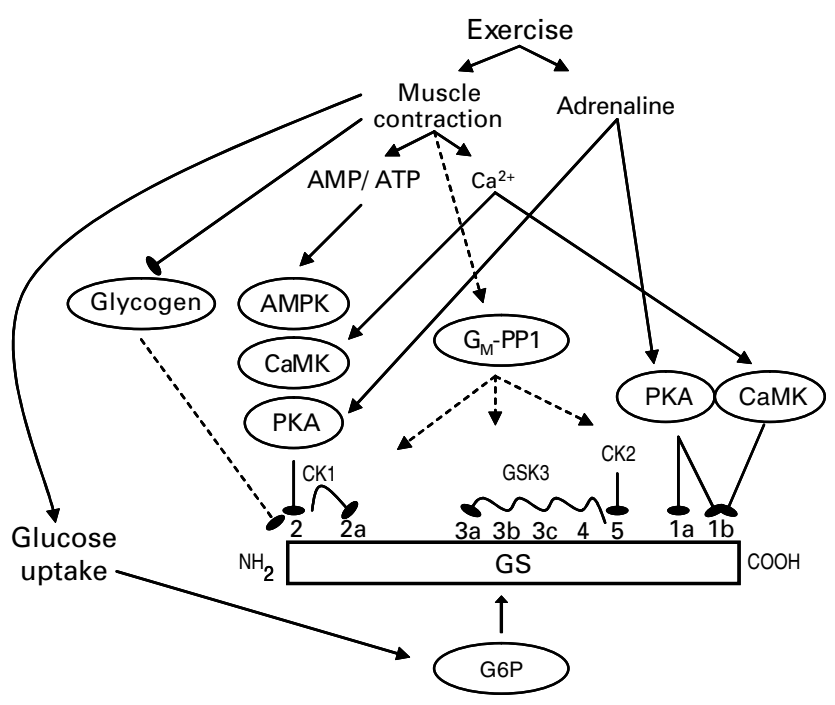

Fig. 1. Regulation of skeletal muscle glycogen synthase (GS) activity and phosphorylation by exercise. Exercise elicits an increase in blood adrenaline levels and muscle contraction leads to changes in intracellular nucleotide and calcium ion levels. Adrenaline activates cAMP-dependent protein kinase (AMPK)/ protein kinase A (PKA), which decreases GS activity by directly phosphorylating site 2 and indirectly phosphorylating site 3 by a mechanism as yet unknown (not indicated). The glycogenassociated protein phosphatase 1 (PP1) subunit $G_{M}$, has been shown to be essential in contraction-activation of GS, but the mechanism by which $G_{M}$ is activated (if at all) by muscle contraction has not been identified. Furthermore, it is not known whether PP1 preferentially dephosphorylates specific sites on GS. Glycogen inhibits GS activity by causing phosphorylation of site 2 on GS, but the mechanism remains elusive. Furthermore, the calcium ion concentration increases in response to muscle contraction and this factor could lead to increased site 2 phosphorylation of GS mediated by calcium ion/calmodulin-dependent kinase (CaMK) or other calcium ion-dependent kinases. Glucose-6-phosphate (G6P) levels often increase during exercise as a result of an increase in glucose uptake and an increase in glycogen breakdown. This change in levels allosterically activates GS. $\leftarrow$, $\bullet$, Activating and inhibiting factors respectively. -----, Effects that are not completely understood. The GS enzyme is shown in a diagrammatic form with indications of the different phosphorylation sites and the primary kinases thought to act on these sites. Hierarchal phosphorylation is indicated on site $2 a$ and the sites 3-4. Sites 2, 2a, 3a and $3 b$ are the sites thought to be most important in the regulation of GS activity. GSK, GS kinase; CK1 and 2, casein kinases 1 and 2. For further detail and references, see p. 233.

\section{Mechanisms by which exercise regulates glucose synthase activity}

Early studies in rodent skeletal muscle have demonstrated that GS activity is increased in response to exercise (Danforth, 1965). However, in response to short-term high-intensity exercise GS activity has actually been observed to be decreased or unchanged during exercise and then rapidly increased after the cessation of exercise (Chasiotis et al. 1982; Jiao et al. 2001). From a narrow viewpoint, this finding suggests that the energy-consuming process of glycogenesis is diminished only when energy is greatly needed elsewhere, and also that this potential waste of energy is allowed during conditions of more moderate exercise. The hypothesis has been put forward that GS activity is influenced by both stimulatory and inhibitory factors during exercise and the consequent effect of exercise is a result of the relative strength of these opposing signals, which may vary according to the mode, intensity and duration of the exercise. This idea is supported by the observation in rodent skeletal muscle, using phospho-sitespecific GS antibodies, that phosphorylation at the $\mathrm{NH}_{2}-$ terminal sites 2 and $2 \mathrm{a}$ and the far $\mathrm{COOH}$-terminal sites $1 \mathrm{a}$ and $1 \mathrm{~b}$ is markedly increased during contraction in vitro (leading to inactivation of GS), whereas phosphorylation on sites $3 \mathrm{a}$ and $3 \mathrm{~b}$ decreases (leading to activation of GS; JN Nielsen, BF Hansen and JFP Wojtaszewski, unpublished results). There seems to be a consensus that exercise and insulin regulate GS activity differentially (Danforth, 1965; Aschenbach et al. 2001; Nielsen et al. 2001). This consensus is based on the observations that the regulation of GS activity by exercise is independent of the muscle insulin receptor (Wojtaszewski et al. 1999a) and phosphatidylinositol 3-kinase, whereas the effect of insulin is not (Marchand-Brustel et al. 1995; Wojtaszewski et al. 1999b). Notably, the stimulatory effects of exercise and insulin are not always additive (Wojtaszewski et al. 1999a), suggesting that the two stimuli, at least in part, affect GS in the same way. This suggestion is also supported by the observation that both insulin and muscle contraction decrease phosphorylation of GS at sites $3 a$ and $3 b$ (Parker et al. 1983; Hojlund et al. 2003; Sakamoto et al. 2003). The way in which exercise may signal the regulation of the activity and phosphorylation of GS will be discussed, with emphasis on some potential kinases (PKA, AMPK, GSK3), phosphatases (protein phosphatase 1; PP1) and other regulatory elements (G6P, glycogen).

\section{Protein kinase A}

As mentioned earlier, GS activity is not always increased, and in some studies even decreased, in response to exercise. Based on in vitro observations activation of several kinases could be responsible for the exercise-induced deactivation of GS in vivo. A decrease in phosphatase activity during exercise would also be able to decrease the activity of GS. Importantly, an inherent response to exercise is an increase in the plasma adrenaline concentration. The cellular action of adrenaline is thought to be mediated through an increase in cAMP, in turn activating PKA. Although sites 2, $1 \mathrm{a}$ and $1 \mathrm{~b}$, but not sites $3 \mathrm{a}$ and $3 \mathrm{~b}$, on GS are phosphorylated by PKA in vitro, phosphorylation of sites $3 \mathrm{a}$ and $3 \mathrm{~b}$ is increased in vivo with adrenaline infusion (for review, see Roach, 2002). This finding indicates that PKA may exert its effect on GS by direct phosphorylation of sites $2,1 \mathrm{a}$ and $1 \mathrm{~b}$ and also by indirect mechanisms affecting phosphorylation of sites $3 \mathrm{a}$ and $3 \mathrm{~b}$ (e.g. through PP1 deactivation, as discussed later). A role for adrenaline in exercise-induced inhibition of GS activity is supported by the following observations: (1) adrenaline infusion in resting human subjects induces a marked drop in skeletal muscle GS activity (Chasiotis et al. $1983 b)$; (2) $\beta$-adrenergic blockade (propranolol infusion) 
abolishes the decrease in GS activity induced by short-term isometric or dynamic exercise (Chasiotis et al. 1983a); (3) in response to electrical stimulation of the sciatic nerve in one leg of the rat, eliciting contractions in one leg while keeping the contra-lateral leg rested, phosphorylation of sites $2,1 \mathrm{a}$ and $1 \mathrm{~b}$ increases in the rested muscle, pointing to the influence of a systemic factor induced by muscle contraction in situ (JN Nielsen, BF Hansen and JFP Wojtaszewski, unpublished results).

\section{5'AMP-activated protein kinase}

Based on the view that AMPK is a sensor of cellular energy charge, turning off ATP-consuming anabolic pathways, it could be proposed that AMPK activation may work to decrease the activity of GS during exercise, slowing down the energy-consuming incorporation of UDP-glucose into glycogen. This mechanism was initially indicated by the observations that site 2 on GS is a substrate of AMPK in vitro (Carling \& Hardie, 1989) and that GS co-immunoprecipitates with AMPK in skeletal muscle (Chen et al. 1999). More recently, several observations have been made that strongly support the notion that AMPK indeed phosphorylates GS at site 2 in vivo. Pharmacological activation of AMPK by 5'-aminoimidazole-4-carboxamide$1-\beta$-D-ribofuranoside (AICAR) in perfused muscles leads to a decrease in GS activity (Wojtaszewski et al. 2002). The change in AMPK activity associated with the $\alpha 2$ AMPK catalytic subunit in response to AICAR is negatively related to the GS activity, and AICAR induces an upward shift in the gel mobility of GS, which is prevented by previous phosphatase treatment (Wojtaszewski et al. 2002). Furthermore, GS obtained from AICAR-perfused (and incubated) rat muscles has increased site 2 phosphorylation, in accordance with the original in vitro findings (SB Jørgensen, JN Nielsen, DG Hardie, BF Hansen, EA Richter and JFP Wojtaszewski, unpublished results). Finally, in muscles from $\alpha 2$ AMPK knock-out mice AICAR does not induce GS deactivation (SB Jørgensen, B Viollet, DG Hardie, BF Hansen, S Vaulont, EA Richter and JFP Wojtaszewski, unpublished results). Thus, AMPK seems to be a GS site 2 kinase. Whether AMPK activation is important for the observed GS site 2 phosphorylation during exercise is currently under investigation.

\section{Glycogen synthase kinase 3}

Apparently, several potential mechanisms exist by which exercise could inhibit GS activity. There still remains the question of how exercise signals to the activation of GS. Although phosphorylation of GS at sites 2 and $2 \mathrm{a}$ is markedly increased at the onset of muscle contraction, phosphorylation of sites $3 \mathrm{a}$ and $3 \mathrm{~b}$ is progressively decreased when muscle contraction is sustained for more than a few minutes, leading to an increase in GS activity (JN Nielsen, BF Hansen and JFP Wojtaszewski, unpublished results). This finding raises the possibility that the activity of a kinase with specificity for sites $3 a$ and $3 b$ would decrease in response to exercise. GSK3 consecutively phosphorylates sites $4,3 \mathrm{a}, 3 \mathrm{~b}$ and $3 \mathrm{c}$ on GS when site 5 is phosphorylated by casein kinase 2 , as discussed earlier. In rodent skeletal muscle GSK3 $\alpha$ and $\beta$ activity and phosphorylation are decreased and increased respectively in response to treadmill exercise, in situ contraction and in isolated muscle contracted in vitro in the absence of systemic factors (Markuns et al. 1999; Sakamoto et al. 2002). Thus, GSK3 could be a potential regulator of GS activity during exercise. However, recent data suggest that although GSK3 activity is decreased with muscle contraction, this decrease does not correlate with the increase in GS activity and phosphorylation of sites $3 \mathrm{a}, 3 \mathrm{~b}, 3 \mathrm{c}$ and 4 on GS (Sakamoto et al. 2003). It has been demonstrated that both contraction and mechanical stretch of isolated rat muscle increase the phosphorylation of GSK3 (leading to inactivation of GSK3), but only contraction decreases the phosphorylation of GS. Furthermore, the phosphatidylinositol 3-kinase inhibitor wortmannin inhibits the contraction and stretch-induced increase in GSK3 phosphorylation but has no effect on phosphorylation of GS at sites $3 a, 3 b, 3 c$ and 4 during these stimuli. The absence of a role for GSK3 in exercise-induced activation of GS is also supported by the observation that GSK3 is not deactivated in human skeletal muscle in response to exercise (Wojtaszewski et al. 2001). Interestingly, recombinant dual-specificity tyrosine phosphorylated and regulated kinases have recently been shown to phosphorylate muscle GS at site 3 (Skurat \& Dietrich, 2003). Whether dual-specificity tyrosine phosphorylated and regulated kinase activity is influenced by exercise in skeletal muscle is presently unknown.

\section{Protein phosphatase 1}

It would be tempting to ascribe the decreased phosphorylation of GS at sites $3 a$ and $3 b$ during exercise to the notion that phosphatase activity directed against these sites is increased with exercise. However, an increase in PP1 activity in response to exercise has never been reported. The primary phosphatase acting on GS is the multisubstrate phosphatase PP1. The regulation of PP1 is complex and has recently been the topic of comprehensive reviews (see Brady \& Saltiel, 2001). In relation to the regulation of GS activity it should be mentioned that GS, glycogen phosphorylase and phosphorylase kinase are all to a variable extent bound directly to glycogen (Cohen, 1978), but several lines of evidence indicate that these enzymes, as well as PP1, are also bound to PP1-targeting subunits that themselves bind to glycogen and act as molecular scaffolds, thereby having a major impact on the regulation of glycogen metabolism. One such targeting subunit that has received considerable attention is the glycogen-associated PP1 subunit $\mathrm{G}_{\mathrm{M}}$ (also termed glycogen-binding regulatory subunit or RGl). Binding of $\mathrm{G}_{M}$ to PP1 enhances dephosphorylation of GS, whereas glycogenolytic agents such as adrenaline cause serine phosphorylation resulting in dissociation and thus inactivation of the $\mathrm{G}_{\mathrm{M}^{-}} \mathrm{PP} 1$ complex (Hiraga \& Cohen, 1986). An essential role for $G_{M}$ in exercise regulation of GS has recently been indicated by a study in which the $G_{M}$ gene was knocked out in mice. After either exercise or in situ muscle contraction GS activity is increased in the wild type but unchanged in the $\mathrm{G}_{\mathrm{M}}$ knock-out mice (Aschenbach et al. 
2001). This finding suggests that $G_{M}$ is essential for the activation of GS after exercise, perhaps through the association with PP1.

\section{Glycogen}

GS activity is negatively feedback-regulated by glycogen (Danforth, 1965; Nielsen et al. 2001), but the mechanism for this effect is largely unknown. The hypothesis has been put forward that glycogen breakdown is a factor involved in GS activation during exercise. It has been observed that the lower GS activity in muscle high in glycogen compared with GS from muscle low in glycogen is associated with elevated phosphorylation at sites 2 and 2a (JN Nielsen and JFP Wojtaszewski, unpublished results), pointing to the notion that glycogen modulates the ability of kinases and phosphatases to act on GS. Previously, it has been observed that protein phosphatase treatment of GS from muscle high in glycogen does not restore GS activity to the same extent as GS obtained from muscle low in glycogen (Wojtaszewski et al. 2002). This finding suggests that events unrelated to phosphorylation also regulate GS activity in response to changes in glycogen content. A potential mechanism could be O-linked N-acetylglucosamine modification, which is a reversible post-translational modification that regulates enzyme activity. Recently, it has been shown in 3T3-adipocytes that exposure to glucosamine induces $\mathrm{O}$-linked $\mathrm{N}$-acetylglucosamine modification of GS, which apparently inactivates the enzyme (Parker et al. 2003). If GS is modified by O-linked $\mathrm{N}$-acetylglucosamine in muscle high in glycogen, this may explain the relative resistance to phosphatase treatment (Wojtaszewski et al. 2002), because it has been shown that purified GS becomes fully de-phosphorylated in response to phosphatase treatment only after removal of O-linked N-acetylglucosamine (Parker et al. 2003). Future studies should address the possibility that the O-linked N-acetylglucosamine modification of GS is changed in response to exercise and/or whether the activity of upstream GS kinases or phosphatases are glycogen sensitive.

\section{Glucose-6-phosphate}

As mentioned earlier, G6P is a direct and indirect stimulator of the activity of GS. Although the direct allosteric effect of G6P in vivo is not detected when GS activity is measured in vitro, it should be considered that G6P may play an important role in regulating GS activity during exercise. Muscle glucose uptake is increased by exercise and this increase often leads to increases in the concentration of G6P levels. Thus, the allosteric activation of GS during exercise could be quite important, as suggested by Bloch et al. (1994). GS activity is also increased in contracting skeletal muscles that have been stimulated to contract in vitro (incubated) or in situ (perfused) in the absence of glucose, keeping G6P levels very low or even unchanged (W Derave, personal communication). This finding suggests that the increase in GS activity induced by exercise is not dependent on an increase in skeletal muscle G6P levels, which is also supported by observations in human muscle cell cultures (Montell et al. 1999).

\section{Summary}

Exercise regulation of GS is characterized by great complexity, because GS is a substrate of multiple upstream enzymes acting on several phosphorylation sites of GS. Furthermore, exercise activates both stimulatory and inhibitory regulators of GS, and the strength of these regulators may vary over time. Although the field is far from being fully elucidated, the following hypothesis has been put forward, based on the literature and the authors' unpublished data. In brief, during the initial stage of exercise, GS activity is inhibited through the increased phosphorylation of site 2 (rendering site $2 \mathrm{a}$ as a substrate for the constitutively-active casein kinase 1) mediated by adrenalineinduced PKA activation, and an increase in AMPK activity. Later in exercise, although the inhibitory signals are undiminished, GS activity is increased through an increase in PP1-mediated dephosphorylation of GS at sites $3 \mathrm{a}$ and $3 \mathrm{~b}$. Nevertheless, major questions remain unanswered. What is the precise mechanism by which exercise signals to PP1, and do PP1 act site-specifically on GS in vivo? What is the role of sites $1 \mathrm{a}$ and $1 \mathrm{~b}$ in the regulation of GS, since phosphorylation of these sites seems to have minor effects on enzyme activity? Finally, it should be considered that O-linked glucosylation might have a role in exercise-regulation of GS activity. Although omitted from the present review, the authors are aware that Ca-sensitive kinases (glycogen phosphorylase kinase, protein kinase $\mathrm{C}, \mathrm{Ca}^{2+}$ /calmodulin-dependent protein kinase) are also potential GS kinases, which may have additional regulatory roles during exercise, and these kinases are also obvious targets for further research in this area.

\section{Acknowledgements}

The authors are supported by a Research \& Technological Development Project (QLG1-CT-2001-01488) funded by the European Commission, grant no. 504-14 from the Danish National Research Foundation, the Media and Grants Secretariat of the Danish Ministry of Culture, Novo-Nordisk Foundation and the Danish Diabetes Association. J.N.N. and J.F.P.W. were supported by the Carlsberg Foundation and a Hallas Møller fellowship from the Novo Nordisk Foundation respectively. The authors acknowledge that in order to keep within the editorial required length it has not always been possible to refer to original papers.

\section{References}

Aschenbach WG, Suzuki Y, Breeden K, Prats C, Hirshman MF, Dufresne SD, Sakamoto K, Vilardo PG, Steele M, Kim JH, Jing SS, Goodyear LJ \& DePaoli-Roach AA (2001) The muscle-specific protein phosphatase $\mathrm{PP} 1 \mathrm{G} / \mathrm{R}$ GL(GM) is essential for activation of glycogen synthase by exercise. Journal of Biological Chemistry 276, 39959-39967.

Bloch G, Chase JR, Meyer DB, Avison MJ, Shulman GI \& Shulman RG (1994) In vivo regulation of rat muscle glycogen resynthesis after intense exercise. American Journal of Physiology 266, E85-E91. 
Brady MJ \& Saltiel AR (2001) The role of protein phosphatase-1 in insulin action. Recent Progress in Hormone Research 56, 157-173.

Carling D \& Hardie DG (1989) The substrate and sequence specificity of the AMP-activated protein kinase. Phosphorylation of glycogen synthase and phosphorylase kinase. Biochimica et Biophysica Acta 1012, 81-86.

Chasiotis D, Brandt R, Harris RC \& Hultman E (1983a) Effects of beta-blockade on glycogen metabolism in human subjects during exercise. American Journal of Physiology 245, E166-E170.

Chasiotis D, Sahlin K \& Hultman E (1982) Regulation of glycogenolysis in human muscle at rest and during exercise. Journal of Applied Physiology 53, 708-715.

Chasiotis D, Sahlin K \& Hultman E (1983b) Regulation of glycogenolysis in human muscle in response to epinephrine infusion. Journal of Applied Physiology 54, 45-50.

Chen Z, Heierhorst J, Mann RJ, Mitchelhill KI, Michell BJ, Witters LA, Lynch GS, Kemp BE \& Stapleton D (1999) Expression of the AMP-activated protein kinase beta1 and beta2 subunits in skeletal muscle. FEBS Letters 460, 343-348.

Cohen P (1978) The role of cyclic-AMP-dependent protein kinase in the regulation of glycogen metabolism in mammalian skeletal muscle. Current Topics in Cellular Regulation 14, 117-196.

Danforth WH (1965) Glycogen synthase activity in skeletal muscle. Interconversion of two forms and control of glycogen synthesis. Journal of Biological Chemistry 240, 588-593.

Hiraga A \& Cohen P (1986) Phosphorylation of the glycogenbinding subunit of protein phosphatase-1G by cyclic-AMPdependent protein kinase promotes translocation of the phosphatase from glycogen to cytosol in rabbit skeletal muscle. European Journal of Biochemistry 161, 763-769.

Hojlund K, Staehr P, Hansen BF, Green KA, Hardie DG, Richter EA, Beck-Nielsen H \& Wojtaszewski JFP (2003) Increased phosphorylation of skeletal muscle glycogen synthase at $\mathrm{NH} 2-$ terminal sites during physiological hyperinsulinemia in type 2 diabetes. Diabetes 52, 1393.

Jiao Y, Shashkin P \& Katz A (2001) A new glycogen synthase activity ratio in skeletal muscle: effects of exercise and insulin. Life Sciences 69, 891-900.

Marchand-Brustel Y, Gautier N, Cormont M \& Van Obberghen E (1995) Wortmannin inhibits the action of insulin but not that of okadaic acid in skeletal muscle: comparison with fat cells. Endocrinology 136, 3564-3570.

Markuns JF, Wojtaszewski JF \& Goodyear LJ (1999) Insulin and exercise decrease glycogen synthase kinase-3 activity by different mechanisms in rat skeletal muscle. Journal of Biological Chemistry 274, 24896-24900.

Montell E, Arias A \& Gomez-Foix AM (1999) Glycogen depletion rather than glucose 6-P increments controls early glycogen recovery in human cultured muscle. American Journal of Physiology 276, R1489-R1495.
Nielsen JN, Derave W, Kristiansen S, Ralston E, Ploug T \& Richter EA (2001) Glycogen synthase localization and activity in rat skeletal muscle is strongly dependent on glycogen content. Journal of Physiology (London) 531, 757-769.

Parker GJ, Lund KC, Taylor RP \& McClain DA (2003) Insulin resistance of glycogen synthase mediated by O-linked $\mathrm{N}$ acetylglucosamine. Journal of Biological Chemistry $\mathbf{2 7 8}$ 10022-10027.

Parker PJ, Caudwell FB \& Cohen P (1983) Glycogen synthase from rabbit skeletal muscle; effect of insulin on the state of phosphorylation of the seven phosphoserine residues in vivo. European Journal of Biochemistry 130, 227-234.

Roach PJ (2002) Glycogen and its metabolism. Current Molecular Medicine 2, 101-120.

Roach PJ \& Larner J (1976) Rabbit skeletal muscle glycogen synthase. II. Enzyme phosphorylation state and effector concentrations as interacting control parameters. Journal of Biological Chemistry 251, 1920-1925.

Roach PJ, Takeda Y \& Larner J (1976) Rabbit skeletal muscle glycogen synthase. I. Relationship between phosphorylation state and kinetic properties. Journal of Biological Chemistry 251, 1913-1919.

Sakamoto K, Aschenbach WG, Hirshman MF \& Goodyear LJ (2003) Akt signaling in skeletal muscle: Regulation by exercise and passive stretch. American Journal of Physiology $\mathbf{2 8 5}$, E1081-E1088.

Sakamoto K, Hirshman MF, Aschenbach WG \& Goodyear LJ (2002) Contraction regulation of Akt in rat skeletal muscle. Journal of Biological Chemistry 277, 11910-11917.

Skurat AV \& Dietrich AD (2003) DYRK Family protein kinases phosphorylates Ser-640 in muscle glycogen synthase. Diabetes 52, 1852.

Wojtaszewski JFP, Higaki Y, Hirshman MF, Michael MD, Dufresne SD, Kahn CR \& Goodyear LJ (1999a) Exercise modulates postreceptor insulin signaling and glucose transport in muscle-specific insulin receptor knockout mice. Journal of Clinical Investigation 104, 1257-1264.

Wojtaszewski JFP, Jorgensen SB, Hellsten Y, Hardie DG \& Richter EA (2002) Glycogen-dependent effects of 5-aminoimidazole-4-carboxamide (AICA)-riboside on AMP-activated protein kinase and glycogen synthase activities in rat skeletal muscle. Diabetes 51, 284-292.

Wojtaszewski JFP, Lynge J, Jakobsen AB, Goodyear LJ \& Richter EA (1999b) Differential regulation of MAP kinase by contraction and insulin in skeletal muscle: metabolic implications. American Journal of Physiology 277, E724-E732.

Wojtaszewski JFP, Nielsen P, Kiens B \& Richter EA (2001) Regulation of glycogen synthase kinase-3 in human skeletal muscle: effects of food intake and bicycle exercise. Diabetes 50, 265-269. 
https://doi.org/10.1079/PNS2004348 Published online by Cambridge University Press 